\title{
Dramatic Response in a Patient with Metastatic Gastric Cancer Using Trifluridine/ Tipiracil after Rapid Disease Progression while on Nivolumab
}

\author{
Kazuki Nozawa ${ }^{a}$ Yukiya Narita ${ }^{a}$ Waki Hosoda ${ }^{b}$ Kei Muro ${ }^{a}$ \\ aDepartment of Clinical Oncology, Aichi Cancer Center Hospital, Nagoya, Japan; \\ ${ }^{b}$ Department of Pathology and Molecular Diagnostics, Aichi Cancer Center Hospital, \\ Nagoya, Japan
}

\section{Keywords}

Gastric cancer · Hyperprogressive disease - Immunotherapy · Nivolumab ·

Trifluridine/tipiracil

\begin{abstract}
The introduction of immune checkpoint inhibitors has redefined the treatment strategy and changed the way tumor assessments are made because of its response pattern. Studies have suggested that initiating chemotherapy after checkpoint inhibitors may have high anti-tumor activity in some cancer types. This response pattern has not been reported in patients with gastric cancer, and particularly for the combination of trifluridine/tipiracil. A 69-year-old man presented at follow-up for metastatic gastric cancer being treated with nivolumab, an antiPD-1 antibody. Computed tomography of the liver showed a rapid 4-fold growth of the metastasis compared with baseline measurements taken while receiving paclitaxel and ramucirumab. It met the definition of a phenomenon called hyperprogressive disease. Nivolumab was discontinued, and he was switched to trifluridine/tipiracil. The liver metastasis was shrunk markedly after 2 months with improvement in his performance status and laboratory data. Sequential therapy starting with immune checkpoint inhibitors followed by cytotoxic agents such as trifluridine/tipiracil may have an apparent efficacy in gastric cancer even though prior immunotherapy demonstrates hyperprogressive disease.

\section{Karger $\stackrel{2}{*}$}




\section{Introduction}

Gastric cancer is the fifth most common cancer and the third leading cause of cancer deaths worldwide. The incidence of gastric cancer is higher in East Asia including Japan than other countries [1]. The 5-year survival rate in Japan is also higher than in other countries, primarily Europe and America because of an early screening and diagnosis system. As a result, there were many cases of gastric cancer which were likely to be cured in Japan. Despite important advances in many facets of gastric cancer knowledge and treatment, survival remains poor, especially in advanced disease.

In the era of immuno-oncology, the introduction of immune checkpoint inhibitors has changed treatment strategies against cancer and improved the survival of patients with different types of advanced cancers, and including those with gastric tumors. Nivolumab, a programmed death-1 (PD-1) inhibiting antibody showed survival benefit compared with placebo in Asian patients with gastric cancer in the 0NO-4538-12 (ATTRACTION-2) trial [2]. Based on this result, nivolumab is one of the standard treatments for advanced gastric cancer as third-line in Asia. Trifluridine is an antineoplastic thymidine-based nucleoside analog, and tipiracil is an agent that enhances the activity of trifluridine. In the TAGS trial, the combination of trifluridine/tipiracil has improved survival in advanced gastric cancer compared to placebo [3]. Trifluridine/tipiracil has become another standard treatment as third line; however, objective response was only $4 \%(95 \% \mathrm{CI}, 2-8)$, and the difference of the median survival time between trifluridine/tipiracil and placebo was 2.1 months. The randomized phase 3 JAVELIN Gastric 300 trial compared the anti-PD-L1 antibody avelumab with usual care chemotherapy as third-line therapy. Results showed that avelumab alone did not improve overall survival (OS) or progression-free survival (PFS) [4]. The KEYNOTE-061 study reported that second-line treatment of advanced gastric cancer with the anti-PD-1 antibody pembrolizumab did not improve OS compared with paclitaxel [5]. These results added to a debate about the effectiveness of immune checkpoint inhibitors and chemotherapy in gastric cancer.

It has been previously reported that the addition of chemotherapy after treatment with immune checkpoint inhibitors improved responses in some cancer types. In non-small cell lung cancer, a 39\% objective response rate (ORR) was reported in single-agent chemotherapy following anti-PD-1 treatment [6]. Kato et al. [7] reported that a 31\% ORR was observed in the patients with gastric carcinoma. Despite these reports, there is no consistent evidence of a positive response pattern.

A novel pattern of tumor response with immune checkpoint inhibitors was reported. The tumor growth rate (TGR) is a tool for estimating an increase in tumor volume of many different cancers. In some reports, treatment with immune checkpointinhibitors resulted in progression of disease that was more rapid than expected. This phenomenon is called hyperprogressive disease (HPD) and is defined as a greater than 2-fold increase in TGR compared to treatment baseline [8]. Another pattern of response exists called pseudoprogression, in which there is an initial increase in tumor size or count before decreasing. Currently, there is no way to distinguish between these two disease patterns of progression occurring from immunotherapy.

In this report, we describe a case of a patient with metastatic gastric cancer who developed rapid disease progression while on nivolumab. Salvage treatment using trifluridine/tipiracil resulted in obvious tumor shrinkage. 


\section{Case Reports in Oncology}

Table 1. Laboratory data at initiation and during treatment with trifluridine/tipiracil

\begin{tabular}{l|l}
\hline Case Rep Oncol 2020;13:1381-1386 \\
\hline DOI: 10.1159/000510405 & $\begin{array}{l}\text { ○ 2020 The Author(s). Published by S. Karger AG, Basel } \\
\text { www.karger.com/cro }\end{array}$ \\
\hline
\end{tabular}

\begin{tabular}{lrrl}
\hline & Initiation & \multicolumn{2}{c}{ During treatment } \\
\hline WBC & 7,820 & 4,600 & $/ \mu \mathrm{L}$ \\
RBC & 392 & 246 & $\times 10^{4} / \mu \mathrm{L}$ \\
Hb & 11.5 & 8.4 & $\mathrm{~g} / \mathrm{dL}$ \\
PLT & 26.1 & 17.5 & $\times 10^{4} / \mu \mathrm{L}$ \\
Alb & 4.1 & 3.6 & $\mathrm{~g} / \mathrm{dL}$ \\
AST & 132 & 27 & $\mathrm{IU} / \mathrm{L}$ \\
ALT & 81 & 17 & $\mathrm{IU} / \mathrm{L}$ \\
ALP & 1,247 & 416 & $\mathrm{U} / \mathrm{L}$ \\
LDH & 2,452 & 162 & $\mathrm{IU} / \mathrm{L}$ \\
Cre & 1.48 & 1.11 & $\mathrm{mg} / \mathrm{dL}$ \\
BUN & 19 & 14 & $\mathrm{mg} / \mathrm{dL}$ \\
T-bil & 0.4 & 0.1 & $\mathrm{mg} / \mathrm{dL}$ \\
CRP & 0.99 & 0.09 & $\mathrm{mg} / \mathrm{dL}$ \\
CEA & 41.4 & 21.6 & $\mathrm{ng} / \mathrm{mL}$ \\
CA19-9 & 240.4 & 56.4 & $\mathrm{U} / \mathrm{mL}$ \\
\hline
\end{tabular}

Alb, albumin; ALP, alkaline phosphatase; ALT, alanine aminotransferase; AST, aspartate transaminase; BUN, blood urea nitrogen; CA19-9, carbohydrate antigen 19-9; CEA, carcinoembryonic antigen; Cre, creatinine; CRP, C-reactive protein; $\mathrm{Hb}$, hemoglobin; $\mathrm{LDH}$, lactate dehydrogenase; PLT, platelet; RBC, red blood cell; T-bil, total-bilirubin; WBC, white blood cell.

\section{Case Report}

A 69-year-old male patient with a history of type 2 diabetes presented to our hospital with a chief complaint of loss of appetite in January 2017. Gastrointestinal endoscopy and biopsy revealed Bormann type 3 gastric cancer with moderately differentiated adenocarcinoma. He underwent total gastrectomy with D3 lymph node dissection. The TNM UICC classification 8th edition was T4aN3aM0, pStage IIIC. Human epidermal growth factor 2 (HER2) was negative and microsatellite instability was stable. The combined positive score of PD-L1 expression was 10\%, and Epstein-Barr encoding region in situ hybridization was negative. After surgery, he received adjuvant combination chemotherapy of oral tegafur/gimeracil/oteracil for 1 year. At his 6-month follow-up, the disease recurred as liver metastasis. Paclitaxel and ramucirumab was started in December 2018, and therapy was switched to nivolumab in August 2019. There was no immune-related adverse events (irAE) while on nivolumab treatment. In November 2019, a computed tomography (CT) scan showed rapid growth of the liver metastasis along with new lesions in the same organ. The estimated TGR of the liver metastasis was 4-fold greater than baseline prior to nivolumab, meeting the definition of HPD. The liver function enzymes aspartate aminotransferase, alanine aminotransferase, and lactate dehydrogenase were elevated during nivolumab treatment. Eastern Cooperative Oncology Group (ECOG) performance status (PS) score was 2 during this period. The patient was then switched to trifluridine/tipiracil dosed at $35 \mathrm{mg} / \mathrm{m}^{2}$ twice daily on days $1-5$, and days 8-12 of a 28-day cycle. His ECOG PS improved from 2 to 0 during trifluridine/tipiracil therapy. The laboratory data throughout the trifluridine/tipiracil therapy period are summarized in Table 1. In the 3 months of trifluridine/tipiracil therapy, carcinoembryonic antigen (CEA) declined from 41.4 to 16.7 $\mathrm{ng} / \mathrm{mL}$, and carbohydrate antigen 19-9 (CA19-9) also decreased from 240.4 to $45.9 \mathrm{U} / \mathrm{mL}$. An interval CT scan in January 2020 showed partial response (Fig. 1). The adverse events of trifluridine/tipiracil therapy included grade 1 neutropenia, nausea, fatigue, and grade 

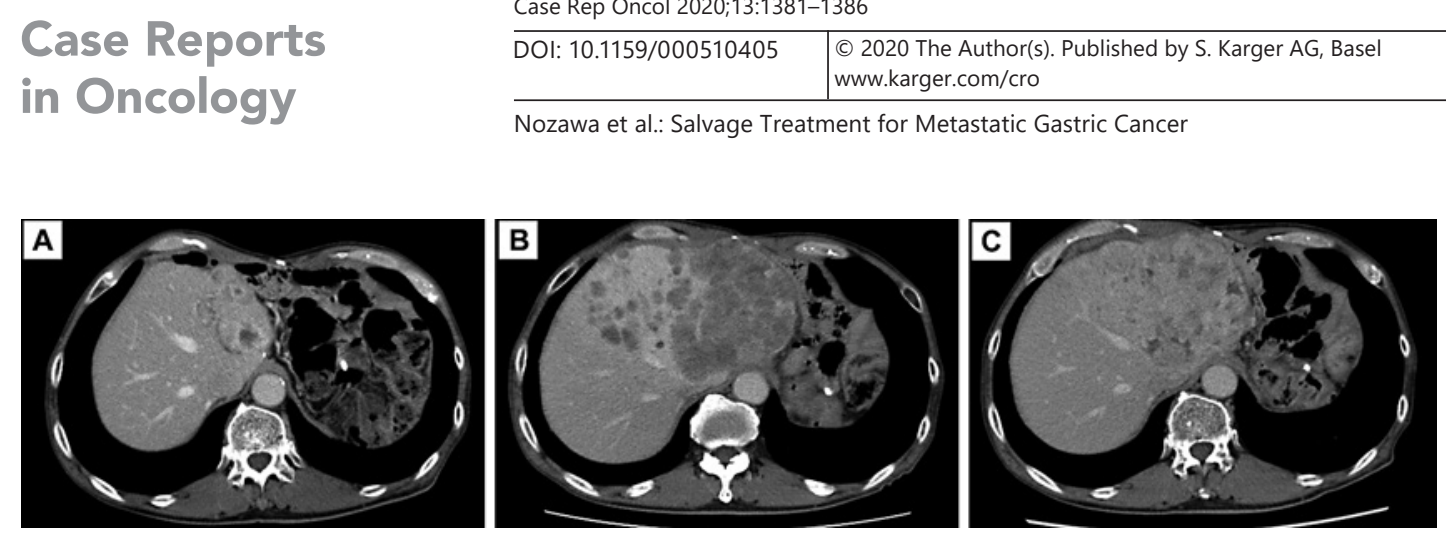

Fig. 1. A Computed tomography (CT) scan in August 2019 after paclitaxel and ramucirumab. B CT scan shows larger lesions in November 2019 after nivolumab. C CT scan shows shrinking lesions in January 2020 while on trifluridine/tipiracil regimen.

2 anemia. There were no serious adverse events or irAEs while on therapy. At the time of writing, the patient continues treatment with trifluridine/tipiracil without serious adverse events or disease progression.

\section{Discussion}

This is the first known report of salvage treatment with trifluridine/tipiracil after nivolumab-induced rapid progression. Increased response rates to salvage chemotherapy after immune checkpoint inhibitor has been reported in some tumor types, especially in nonsmall cell lung cancer. A multicenter retrospective observational cohort study by Kato et al. [9] compared the anti-tumor effects of chemotherapy after PD-1 inhibitor treatment with chemotherapy alone. A significantly higher ORR was reported with sequential therapy compared with chemotherapy alone (18.9 vs. $11.0 \%$, respectively). However, there was no significant difference in PFS and OS.

There may be immunomodulatory effects from trifluridine/tipiracil monotherapy after nivolumab treatment. In vivo results from Yan et al. [10] showed that increased CX3CR1+CD8+ $\mathrm{T}$ cells were associated with the outcome of chemotherapy after immune checkpoint inhibitors. That study reported that CXCR1+ granzyme B + CD8+ T cells increased more often after sequential therapy of anti-PD-1 antibody to chemotherapy than either concurrent treatment, or chemotherapy alone, or anti-PD-1 antibody alone. An ongoing multicenter prospective study is evaluating the efficacy and safety in chemotherapy after progressive disease of nivolumab therapy for metastatic cancer in Japan (UMIN000032182: https://upload.umin. ac.jp/cgi-open-bin/ctr_e/ctr_view.cgi?recptno=R000036615). This study should provide robust evidence on the use of chemotherapy after immune checkpoint inhibitor.

Anti-PD1 antibodies were thought to still be at therapeutic levels at the time of follow-on chemotherapy initiation, given the long half-life of the biologic (i.e., 12-20 days, solid tumors) [11]. The short interval between anti-PD-1 antibody discontinuation and initiation of chemotherapy may have an overlapping effect similar to concurrent therapy. In vivo studies have shown that trifluridine/tipiracil had synergistic anti-tumor efficacy in combination with antiPD-1 antibodies in microsatellite stable (MSS) type murine colorectal cancer cells [12]. However, in a phase 2 study by Patel et al. [13], no clinical benefit was observed for trifluridine/tipiracil plus nivolumab treatment with MSS metastatic colorectal cancer.

The debate about whether nivolumab or trifluridine/tipiracil is more effective in gastric cancer is not yet resolved. One of the answers could be the number of patients receiving sequential treatment. In the TAGS and ATTRACTION-2 trials, 25 and 47\%, respectively, of 
patients with progression received subsequent systemic anticancer therapies [2, 3]. Nivolumab may be the better choice for third-line treatment in gastric cancer because of the higher response rate and proportion of subsequent therapy than trifluridine/tipiracil. In addition, patients with a complete or partial response in the nivolumab group were reported to have excellent median OS (26.6 months) [14].

Overcoming HPD with trifluridine/tipiracil is an important aspect of this case. Several studies reported that HPD during anti-PD-1/PD-L1 therapy was identified in 6-29\% of various cancer types, including melanoma, lung, head and neck, renal, and colorectal cancer. Sasaki et al. [15] reported that $21 \%$ of gastric cancers treated with PD-1 or PD-L1 antibodies developed HPD. There were no differences in HER2, PD-L1, DNA mismatch repair, and Epstein-Barr virus statuses between the HPD and the non-HPD group. The precise mechanism of HPD after immune checkpoint inhibitor is not clear, neither is it for pseudoprogressive disease. Currently, there is no consensus on how to treat patients with HPD.

Sequential therapy starting with immune checkpoint inhibitors followed by chemotherapy was reported to increase adverse events. The TAGS trial reported grade 3 or worse adverse events in $80 \%$ of patients [3]. In this case, there was no serious adverse event or irAE reported during trifluridine/tipiracil therapy, which is continuing for more than 5 months as of this writing. On the other hand, developing chemotherapy-induced neutropenia was reported to improve the survival of colorectal cancer patients treated with trifluridine/tipiracil in the RECOURSE and J003 trials; however, the present case did not have neutropenia during trifluridine/tipiracil therapy [16]. Trifluridine/tipiracil after immune checkpoint inhibitor appears to be relatively well-tolerated in this case.

\section{Conclusion}

We reported a case of remarkable tumor response to trifluridine/tipiracil with no serious adverse events after rapid disease progression while receiving nivolumab. Published evidence indicates that chemotherapy after immune checkpoint inhibitors may enhance the anti-tumor activity. We described a challenging treatment with trifluridine/tipiracil after exposure to immune checkpoint inhibitor and rapid progression. It would be informative to have further studies that identify gastric cancer patients who are most likely to respond to this regimen.

\section{Statement of Ethics}

Written informed consent for publication of the case was obtained.

\section{Conflict of Interest Statement}

Y.N. reports grants and personal fees from Ono Pharmaceutical Co. Ltd. and Bristol-Myers Squibb, personal fees from Eli Lilly, Yakult Honsha, Daiichi Sankyo, and Taiho, outside the submitted work. K.M. reports grants and personal fees from Ono, grants and personal fees from Sanofi, grants from Daiichi Sankyo, grants from Parexel International, grants from Shionogi Pharma, grants from Sumitomo Dainippon, grants from MSD, grants from Pfizer, grants from Mediscience Planning, grants from Solasia Pharma, personal fees from Eli Lilly, personal fees from Chugai, personal fees from Takeda, personal fees from Taiho, personal fees from Bristol-Myers Squibb, personal fees from Bayer, outside the submitted work.

\section{Karger'k}




\section{Case Reports in Oncology}

Case Rep Oncol 2020;13:1381-1386

Funding Sources

No financial support was used for this case report.

\section{Author Contributions}

K. Nozawa searched the literature and wrote the manuscript. Y. Narita and K. Muro conceived and edited the manuscript. W. Hosoda gave input about pathology. All authors have read and approved the final manuscript.

\section{References}

1 Bray F, Ferlay J, Soerjomataram I, Siegel RL, Torre LA, Jemal A. Global cancer statistics 2018: GLOBOCAN estimates of incidence and mortality worldwide for 36 cancers in 185 countries. CA Cancer J Clin. 2018 Nov;68(6): 394-424.

2 Kang YK, Boku N, Satoh T, Ryu MH, Chao Y, Kato K, et al. Nivolumab in patients with advanced gastric or gastrooesophageal junction cancer refractory to, or intolerant of, at least two previous chemotherapy regimens (ONO-4538-12, ATTRACTION-2): a randomised, double-blind, placebo-controlled, phase 3 trial. Lancet. 2017 Dec 2;390(10111):2461-71.

3 Shitara K, Doi T, Dvorkin M, Mansoor W, Arkenau H-T, Prokharau A, et al. Trifluridine/tipiracil versus placebo in patients with heavily pretreated metastatic gastric cancer (TAGS): a randomised, double-blind, placebocontrolled, phase 3 trial. Lancet Oncol. 2018 Nov;19(11):1437-48.

4 Bang YJ, Ruiz EY, Van Cutsem E, Lee KW, Wyrwicz L, Schenker M, et al. Phase III, randomised trial of avelumab versus physician's choice of chemotherapy as third-line treatment of patients with advanced gastric or gastrooesophageal junction cancer: primary analysis of JAVELIN Gastric 300. Ann Oncol. 2018 Oct 1;29(10):205260.

5 Shitara K, Özgüroğlu M, Bang YJ, Di Bartolomeo M, Mandalà M, Ryu MH, et al. Pembrolizumab versus paclitaxel for previously treated, advanced gastric or gastro-oesophageal junction cancer (KEYNOTE-061): a randomised, open-label, controlled, phase 3 trial. Lancet. 2018 Jul 14;392(10142):123-33.

6 Schvartsman G, Peng SA, Bis G, Lee JJ, Benveniste MFK, Zhang J, et al. Response rates to single-agent chemotherapy after exposure to immune checkpoint inhibitors in advanced non-small cell lung cancer. Lung Cancer. 2017 Oct;112:90-5.

7 Kato K, Narita Y, Mitani S, Honda K, Masuishi T, Taniguchi H, et al. Efficacy of cytotoxic agents after progression on Anti-PD-(L)1 antibody for pre-treated metastatic gastric cancer. Anticancer Res. 2020 Apr;40(4):2247-55.

8 Champiat S, Dercle L, Ammari S, Massard C, Hollebecque A, Postel-Vinay S, et al. Hyperprogressive disease is a new pattern of progression in cancer patients treated by anti-PD-1/PD-L1. Clin Cancer Res. 2017 Apr 15; 23(8):1920-8.

9 Kato R, Hayashi H, Chiba Y, Miyawaki E, Shimizu J, Ozaki T, et al. Propensity score-weighted analysis of chemotherapy after PD-1 inhibitors versus chemotherapy alone in patients with non-small cell lung cancer (WJOG10217L). J Immunother Cancer. 2020 Feb;8(1).

10 Yan Y, Cao S, Liu X, Harrington SM, Bindeman WE, Adjei AA, et al. CX3CR1 identifies PD-1 therapy-responsive CD8+ T cells that withstand chemotherapy during cancer chemoimmunotherapy. JCI Insight. 2018 Apr 19;3(8).

11 Brahmer JR, Drake CG, Wollner I, Powderly JD, Picus J, Sharfman WH, et al. Phase I study of single-agent antiprogrammed death-1 (MDX-1106) in refractory solid tumors: safety, clinical activity, pharmacodynamics, and immunologic correlates. J Clin Oncol. 2010 Jul 1;28(19):3167-75.

12 Suzuki N, Tsukihara H, Nakagawa F, Kobunai T, Takechi T. Synergistic anticancer activity of a novel oral chemotherapeutic agent containing trifluridine and tipiracil in combination with anti-PD-1 blockade in microsatellite stable-type murine colorectal cancer cells. Am J Cancer Res. 2017;7(10):2032-40.

13 Patel MR, Falchook GS, Hamada K, Makris L, Winkler RE, Gordon GS, et al. Results of a phase II study evaluating trifluridine/tipiracil plus nivolumab in patients with heavily pretreated microsatellite-stable (MSS) metastatic colorectal cancer (mCRC). J Clin Oncol. 2019;37(8_suppl):48. 10.1200/JCO.2019.37.8_suppl.48.

14 Chen LT, Satoh T, Ryu MH, Chao Y, Kato K, Chung HC, et al. A phase 3 study of nivolumab in previously treated advanced gastric or gastroesophageal junction cancer (ATTRACTION-2): 2-year update data. Gastric Cancer. 2020 May;23(3):510-9.

15 Sasaki A, Nakamura Y, Mishima S, Kawazoe A, Kuboki Y, Bando H, et al. Predictive factors for hyperprogressive disease during nivolumab as anti-PD1 treatment in patients with advanced gastric cancer. Gastric Cancer. 2019 Jul;22(4):793-802.

16 Yoshino T, Cleary JM, Van Cutsem E, Mayer RJ, Ohtsu A, Shinozaki E, et al. Neutropenia and survival outcomes in metastatic colorectal cancer patients treated with trifluridine/tipiracil in the RECOURSE and J003 trials. Ann Oncol. 2020 Jan;31(1):88-95. 